

Conférence Méditerranéenne Côtière et Maritime EDITION 3, FERRARA, ITALIA (2015)

Coastal and Maritime Mediterranean Conference

Disponible en ligne - http://www.paralia.fr - Available online

\title{
Distribution des sédiments fins depuis le bassin versant de l'oued Isser à son embouchure en Méditerranée (Boumerdès, Algérie)
}

\author{
Mohamed BOUHMADOUCHE ${ }^{1}$
}

\begin{abstract}
1. Laboratoire Géo-environnement, Fac. des Sciences de la Terre et de l'Aménagement du Territoire. Université Houari Boumedienne, BP 32 El-Alia, Alger, Algérie. mbouhamadouche@gmail.com
\end{abstract}

\section{Résumé}

Le matériel fluviatile de l'oued (rivière) Isser fourni à la mer présente un fort taux en particules fines originaire du bassin versant de l'oued Isser. Ce dernier étant riche en formations marneuses, marno-calcaires et schisteuses, il n'est donc pas étonnant de constater que les argiles sont abondantes dans la baie de Zemmouri, puisque l'oued Isser est le principal vecteur d'apport véhiculant les sédiments détritiques du domaine continental vers le domaine marin.

La granulométrie de la fraction lutitique suit une évolution progressive depuis l'amont (pont de la Traille) jusqu'à l'embouchure avec un indice d'évolution $\mathrm{N}$ décroissant passant ainsi du faciès sublogarithmique au faciès hyperbolique.

L'oued Isser présente un faible pourcentage sableux (9,89\% en moyenne) d'où un faciès argilo-silteux. Les argiles sont constituées essentiellement de La kaolinite, en plus fortes teneurs (48,1\% en moyenne), de l'illite (22,15\%), d'interstratifiés et autres sédiments détritiques (18,35\%) et de Chlorite (11,4\%).

Mots clés : Oued Isser, Méditerranée, Sédiment fins, Minéraux argileux, Facies, Granulométrie.

\section{Introduction}

Ce travail constitue une contribution à un thème de recherche orienté vers la détermination des phénomènes géologiques géomorphologiques et sédimentologiques en zone côtière. La zone d'étude se trouve sur la cote algérienne au centre à $60 \mathrm{~km}$ à l'est d'Alger (figure 1).

Cette étude a permis de constater une importante diminution des apports fluviatiles de l'oued Isser et cette diminution est plus marquée pour les apports solides ceci est dû principalement à un assèchement du climat ayant une influence directe sur les débits et par conséquent sur les apports sédimentaires.

L'autre cause très importante est la fermeture de cette rivière par la construction du barrage de Beni Amrane. Néanmoins l'étude sédimentologique a permis de cerner la nature des sédiments débités par l’Isser, leurs modalités de dépôt et les différents mécanismes hydro-sédimentaires affectant la région. 
Côtes méditerranéennes menacées :

Risques et défis dans le contexte du changement climatique

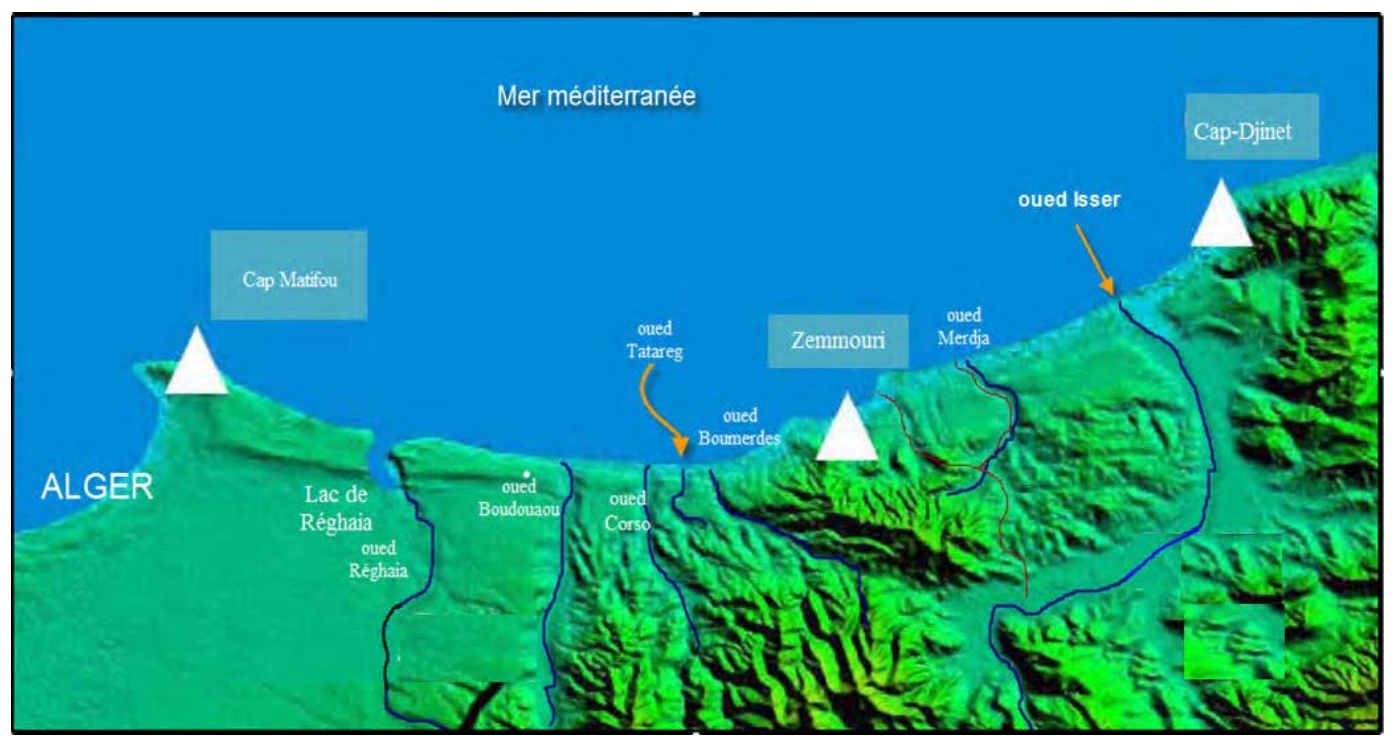

Figure 1. Situation géographique de la zone d'étude.

\section{Méthodologie}

Les prélèvements en mer ont été effectués par l'utilisation d'une benne preneuse de type Van Veen. Les indices d'évolution ont été calculés suivant la méthode dite de "Rivière" (RIVIERE, 1977). alors que les minéraux argileux ont été déterminés par diffraction X avec un appareil de marque Phillips type PW 1710.

\section{Discussion}

\subsection{Caractères sédimentologiques de la fraction fine}

Nous avons essayé de déterminer les modalités de transport et de dépôt de la fraction lutitique dans et autour (zone marine) de oued Isser pour tenter de déceler un éventuel ancien prodelta ou du moins un envasement précoce suivant certains auteurs (ALOISI et al., 1975).

De ce fait nous avons pris des échantillons ciblés positionnés comme le montre la figure 2 et nous avons remarqué que :

- Les courbes granulométriques des échantillons cités font apparaître 2 aires à faciès logarithmiques et sublogarithmique, l'une située au Sud-Ouest, l'autre au Nord-Est (figure 2).

- Les faciès paraboliques et hyperboliques (facies de sédiment profond) s'intercalent suivant une ligne allant de l'échantillon A2 à l'Est jusqu'au canyon de Zemmouri (échantillon Z4 sur la figure 2). Dans l'oued Isser nous avons déterminé deux faciès : un faciès sublogarithmique et un faciès hyperbolique. 


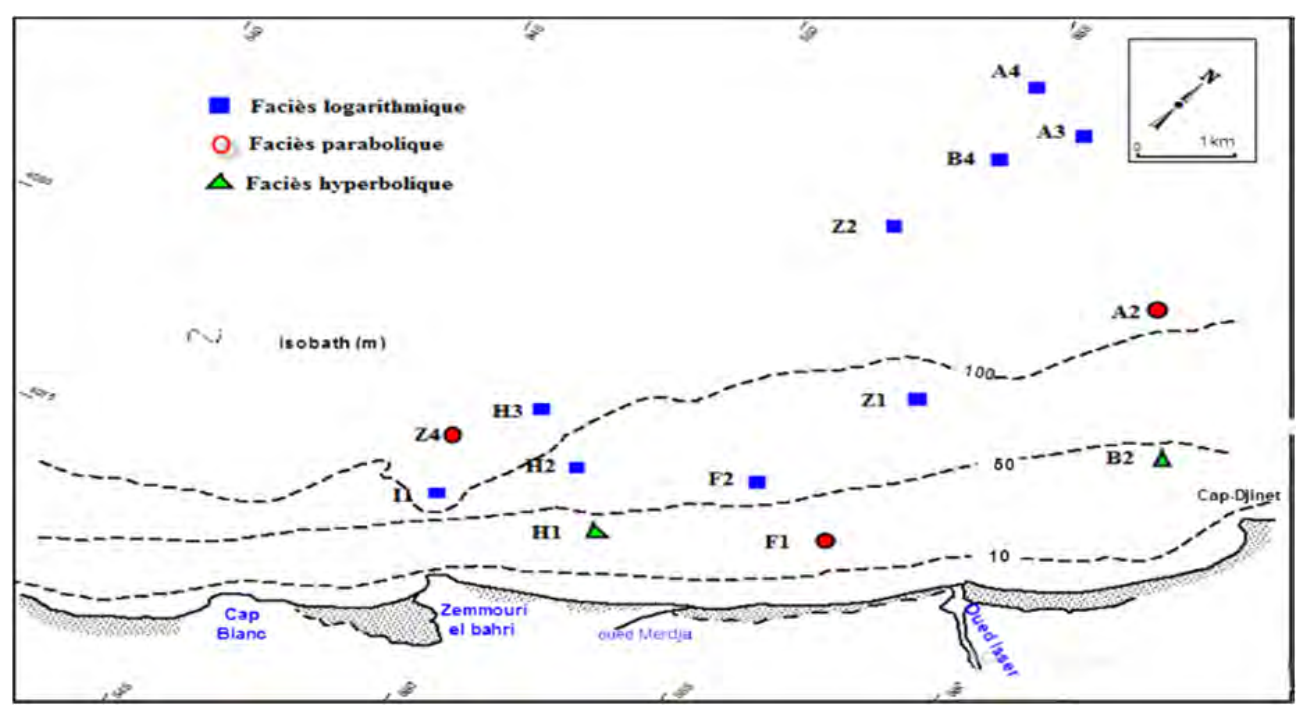

Figure 2. Domaine marin de l'Isser types de facies et indice d'évolution.

\subsubsection{Domaine fluviatile}

Les faciès observés dans l'oued Isser sont :

a) Faciès sublogarithmique. La courbe granulométrique représentative en coordonnées semi-logarithmiques de l'échantillon du Pont de la Traille (0I1) apparaît comme le terme ultime de l'évolution de sédiments transportés par les courants et déposés par excès de charge lorsque la vitesse diminue. De même, l'échantillon situé à l'amont de l'embouchure de l'oued Isser (012) présente un faciès sublogarithmique qui correspond à une suspension dégradée au sens de Passega. Le dépôt s'est fait par excès de charge lorsque l'énergie diminue.

b) Faciès hyperbolique. A l'embouchure, le faciès granulométrique est hyperbolique. La courbe cumulative semi-logarithmique correspondante présente la concavité vers le bas. Son évolution est régulière : témoignant d'un faciès caractéristique des dépôts vaseux de l'embouchure (figure 3, échantillon. OI3).

\subsubsection{Domaine marin}

Les faciès dans le domaine marin sont :

a) Faciès logarithmique et sublogarithmique. Ce faciès a été observé pour les échantillons situés à des profondeurs comprises entre - 40m et - 70m dans l'aire Ouest des faciès logarithmiques (figure 2, Ech : Z1 ; F2 ; B2 et H1) et ceux du talus continental entre - 100 et - 200m (ech.H3), ainsi qu'au-delà de ce dernier, à 400m de profondeur (ech.A4).

b) Faciès hyperbolique. L'indice moyen d'évolution granulométrique étant inférieur à zéro, le faciès granulométrique est alors hyperbolique Ces courbes ont été observées dans les échantillons: Z4, A2, et F1. 
Côtes méditerranéennes menacées :

Risques et défis dans le contexte du changement climatique

c). Faciès parabolique. L'indice d'évolution granulométrique étant supérieur à zéro, le faciès granulométrique est donc parabolique (figure 2, B2 et H1).Tous ces échantillons sont détaillés au tableau 1.

\subsection{Répartition de la fraction minérale argileuse $(<2 \mu \mathrm{m})$}

\subsubsection{Domaine marin}

Les minéraux argileux sont classiquement utilisés comme marqueurs hydrodynamiques (Aloïsi, 1986).

La fraction argileuse est constituée de minéraux argileux tels que, kaolinite, illite chlorite, organisées suivant un ordre de grandeur décroissant.

La répartition de ces minéraux sur le plateau continental Est-Algérois (baie de Zemmouri) est très significative, étant donné la présence de deux facteurs importants : les apports de l'oued Isser drainant des formations néogènes issues de son bassin versant et la proximité du massif volcanique du Cap-Djinet.

\subsubsection{Domaine fluviatile}

La distribution des argiles dans le bassin versant de l'Isser est comme suit :

a) Kaolinite. C'est le minéral argileux le plus fréquent dans les sols des pays tempérés (CAILLERE et al. 1982). Elle présente des teneurs peu variables dans l'oued Isser (48\% en moyenne), c’est le minéral argileux dominant.

b) Illite. Sa répartition dans le lit de l'oued Isser (figure 3) est homogène, 22\% en moyenne, comme la kaolinite, l’illite est retrouvée également sous climat humide au cours de la décomposition des roches acides riches en feldspath (CAILLERE et al., 1982).

c) Chlorite. Moins fréquente que l'Illite et la Kaolinite, elle présente des teneurs de 13\% (OI2), ces teneurs diminuent sensiblement au niveau de l'embouchure marquant la valeur de 7,9\% (OI1) (figure 3). La Chlorite est un minéral typique du métamorphisme. Elle pourrait donc provenir de toutes les formations métamorphiques existantes dans le bassin de l'Isser, entre autres le massif schistocristallin de Krachna qui comprend des schistes à chlorite. 


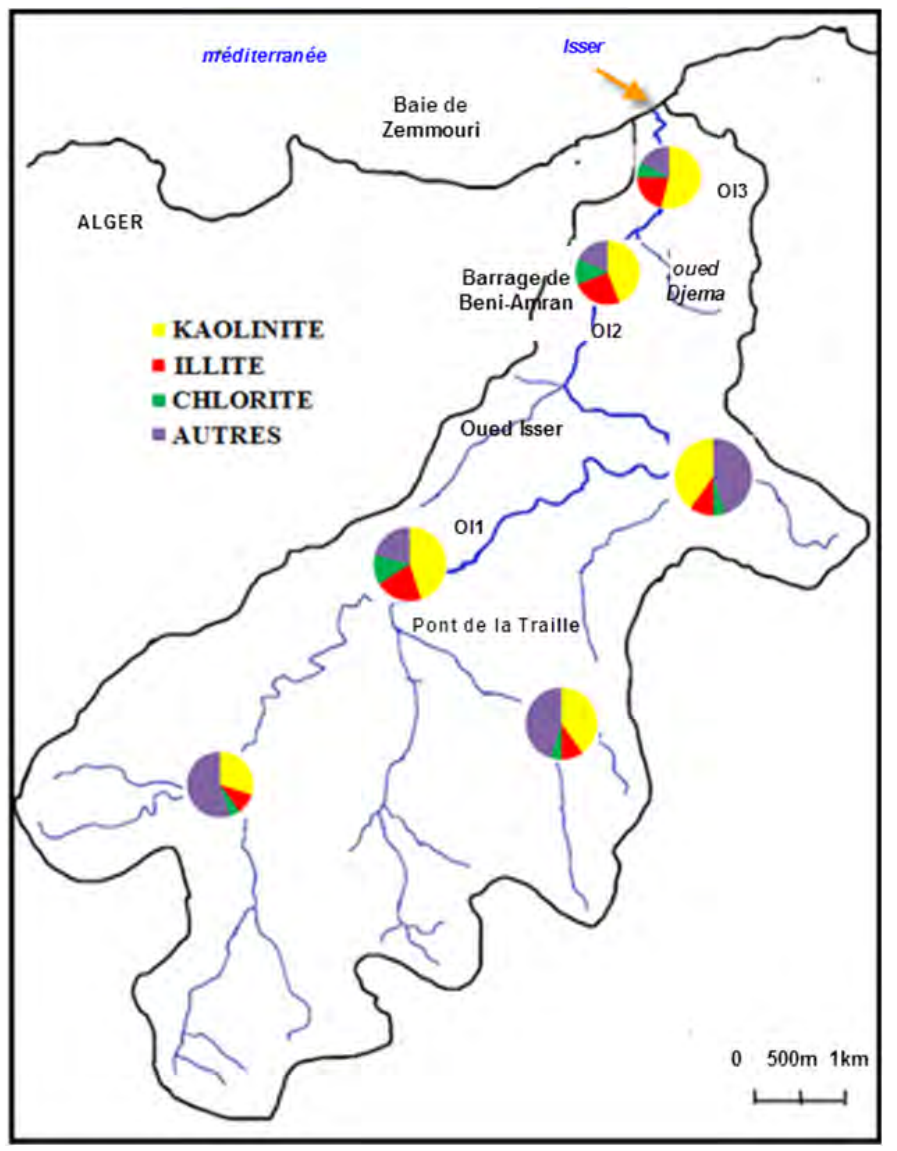

Figure 3. Proportions des minéraux argileux dans l'oued Isser et son bassin versant.

\section{Conclusion}

Notre étude met en évidence la dominance de la kaolinite dans le cortège argileux, les résultats confirment les travaux de CHAMLEY (1971) et LECLAIRE (1972). Les pourcentages moyens des minéraux argileux sont les suivant ; Kaolinite 42,89\%, l’illite 23,66\%, la Chlorite 17,83\% et les interstratifiés 15,62\%.(tab.1)

L'existence d'un envasement précoce en face de l'oued Isser pourrait être argumentée par les facteurs suivants :

- Pourcentages très élevés en lutites (entre75\% et 90\%) à des profondeurs faibles, répartis au voisinage de l'oued Isser.

- Forts taux en silts fins en face de l'oued Isser avec des teneurs supérieures à30\%.

- Forts taux en silts fins en face de l'oued Isser (figure 3 et tableau 1), présentant des teneurs supérieures à30\%.

- Faciès hyperbolique ne concernant qu'un seul échantillon (Fl) proche de l'embouchure et situé à $-20 \mathrm{~m}$ de profondeur de la côte Ouest (tableau 1, Particulièrement pour la marge algérienne, les minéraux argileux permettent de mettre en évidence le changement climatique survenu en Afrique du Nord autour de -7500 à -7000 ans PB passant de l'illite dominante à la kaolinite. (PAUC, 1991). 
Côtes méditerranéennes menacées :

Risques et défis dans le contexte du changement climatique

Tableau 1.Pourcentage des différentes classes granulométriques et type de faciès.

\begin{tabular}{|c|c|c|c|c|c|c|c|}
\hline prélèvement & profondeur moyenne (m) & \% lutites & \% silts grossiers & \% silts fins & \% Argiles & Indices d'évolution (N) & Types de faciès \\
\hline OI1 & - & 72.83 & 4.1 & 23.9 & 60 & -0.04 & sublogarithmique \\
\hline OI2 & - & 98.25 & 3.2 & 29 & 55 & -0.05 & sublogarithmique \\
\hline OI3 & - & 99.25 & 3 & 32 & 60 & -0.15 & hyperbolique \\
\hline Z1 & 57 & 92.52 & 5.5 & 40 & 39 & -0.06 & sublogarithmique \\
\hline Z2 & 200 & 31.14 & 6.5 & 27.6 & 56 & 0.13 & sublogarithmique \\
\hline Z4 & 300 & 98.45 & 0 & 19 & 78 & -0.11 & hyperbolique \\
\hline A2 & 63 & 97.12 & 1 & 34.2 & 60 & -0.08 & hyperbolique \\
\hline A3 & 95 & 39.26 & 5 & 28.7 & 55 & -0.03 & sublogarithmique \\
\hline A4 & 400 & 99.44 & 6 & 38.4 & 54.5 & 0 & logarithmique \\
\hline H1 & 22.5 & 19.18 & 8 & 20 & 58 & -1.15 & hyperbolique \\
\hline H2 & 72.5 & 91.59 & 9 & 25 & 47 & -0.01 & sublogarithmique \\
\hline H3 & 160 & 96.61 & 4.5 & 30 & 55.6 & 0 & logarithmique \\
\hline B2 & 63 & 40.93 & 3 & 24.4 & 60 & 0.17 & parabolique \\
\hline B4 & 200 & 83.56 & 4 & 28 & 59 & -0.04 & sublogarithmique \\
\hline F1 & 20 & 61.87 & 7.5 & 31 & 42 & -0.1 & hyperbolique \\
\hline F2 & 64 & 87.44 & 6.5 & 28.2 & 48 & -0.07 & sublogarithmique \\
\hline I1 & 40 & 34.62 & 5 & 28 & 54 & 0.02 & sublogarithmique \\
\hline
\end{tabular}

\section{Références bibliographiques}

ALOISI J.C., MONACO. A., PAUC H. (1975). Mécanisme de la formation des prodeltas dans le Golfe du Lion. Bull. Insti. Bassin d'Aquitaine., Vol. 18, pp 3-12.

CAILLERE S., HENIN S., RAUTUREAU M. (1982). Minéralogie des argiles. Ed. Masson, Paris, Tomes 1 (184 p) et 2 (189 p).

CHAMLEY H. (1971). Recherche sur la sédimentation argileuse en Méditerranée, Thèse Université de Marseille, 401p.

LECLAIRE L. (1972). La sédimentation holocène sur le versant méridional du bassin Algéro-Baléares. Thèse doctorat d'état, Fac. des Sci. Paris, 382 p.

PAUC H. (1991). La nature minéralogique des apports en suspension sur la marge algérienne et leurs relations avec les sédiments. $3^{\text {ème }}$ Congrès Français de Sedimentologie, Brest; 18,19, 20 novembre 1991.

RIVIERE A. (1977). Méthodes granulométriques : techniques et interprétations ; Ed. Masson Paris, 170 p. 